\title{
Enrichment and identification of differentially expressed genes in hepatocellular carcinoma stem-like cells
}

\author{
JIANG LI $^{1^{*}}$, KAI LIU ${ }^{2 *}$, YUEHONG SHENG ${ }^{3 *}$, QIN ZHANG ${ }^{1}$, LEI CHEN ${ }^{1}$, \\ HAIHUA QIAN $^{1}$, HONGPING WU ${ }^{1}$ and CHANGQING SU ${ }^{1}$ \\ Departments of ${ }^{1}$ Molecular Oncology, National Center for Liver Cancer, ${ }^{2}$ Biliary Tract Surgery IV and \\ ${ }^{3}$ Minimal Invasion Therapy, Eastern Hepatobiliary Surgery Hospital, Navy Military Medical University, \\ Shanghai 200438, P.R. China
}

Received March 30, 2020; Accepted August 18, 2020

DOI: 10.3892/ol.2020.12162

\begin{abstract}
Cancer stem cells are considered to be tumor-initiating cells. To explain the initiation or progression of hepatocellular carcinoma (HCC), we previously established a culture system that may enrich hepatic cancer stem-like cells (HCSCs). However, the regulatory mechanisms by which HCSCs acquire stem cell properties remain unclear. In the present study, three pairs of HCSCs and case-matched human HCC cells were analyzed by high-throughput screening, and novel biomarkers and pathways for the regulation of HCSCs were identified. The results led to the identification and stratification of 406 differentially expressed genes (DEGs), among which 73 GO terms were found to be significantly associated with DEGs in HCSCs, and only complement and coagulation cascade pathways were identified during the development of HCSCs. By combining the results of the Gene Ontology and Kyoto Encyclopedia of Genes and Genomes analyses, it was revealed that 7 genes were downregulated in the complement and coagulation cascade pathways, and 7 miRNAs were predicted to target several downregulated genes involved in these pathways. The results may contribute toward hepatic cancer stem cell studies and novel drug research for HCC treatment.
\end{abstract}

\section{Introduction}

Hepatocellular carcinoma (HCC) is the most common reported malignancy and the second leading cause of all

Correspondence to: Professor Changqing $\mathrm{Su}$, Department of Molecular Oncology, National Center for Liver Cancer, Eastern Hepatobiliary Surgery Hospital, Navy Military Medical University, 225 Changhai Road, Shanghai 200438, P.R. China

E-mail: suchangqing@gmail.com

*Contributed equally

Key words: Hepatocellular carcinoma, cancer stem-like cells, high-throughput screening, differentially expressed genes, signaling pathway cancer-associated mortalities (1). At present, surgical intervention is considered the first option for treating patients with HCC. However, their prognosis remains poor due to recurrence, and resistance to chemotherapy and radiotherapy makes tumor recurrence almost inevitable (2-5).

In attempting to explain HCC carcinogenesis, the theory of cancer stem cells (CSCs), also referred to as tumor-initiating cells (TICs), has attracted wide attention. Experimental evidence supported by clinical observations has highlighted the importance of the association of tumor drug resistance-related properties in HCC with CSCs (6-8). In our previous study, a cell culture system allowing the enrichment of hepatic stem-like cancer cells (HCSCs) from HCC cell lines and human primary HCC tissues was established. HCSCs were demonstrated to show stem cell properties, including the overexpression of stem cell markers, acquisition of epithelial-mesenchymal transition (EMT), drug resistance, and increased tumor-initiating capabilities (9).

Over the past two decades, several well-accepted stem cell surface markers have been exploited in HCSCs, including CD133 (7), CD90 (10) and EpCAM (11). However, the regulatory mechanism by which HCSCs acquire the ability to resist drugs and be efficiently transplanted remains unclear. Therefore, high-throughput transcriptomic sequencing is essential for better understanding the cellular characteristics and signaling pathways of HCSCs involved in tumor initiation or progression (12).

The present study developed a serum-free cell culture system that allows the enrichment and expansion of HCSCs and maintains their stem cell properties with the most important characteristic of tumor initiation ability. The HCSCs exhibit the upregulation of CSC biomarkers, including CD90, the enhancement of EMT properties, the potential of self-renewal and invasion, and resistance to chemotherapeutics. Furthermore, the tumor-initiating ability of the HCSCs is significantly enhanced (9). Therefore, the cell culture system developed in our previous study confirmed the reliability of HCSCs sourced from HCC cell lines. Subsequent research was performed via small RNA high-throughput sequencing in HCSCs, and the integrated miRNA and mRNA data preliminarily clarified the regulatory networks of HCSCs. 
In the present study, to further profile the enriched signatures in HCSCs, the high-throughput screening of 3 pairs of HCSCs and case-matched human HCC cells was performed. The forthcoming integrated miRNA and mRNA data will reveal HCSC regulatory networks according to their properties associated with tumor initiation and chemotherapeutic resistance. The present study identifies novel biomarkers and pathways for the regulation of HCSCs.

\section{Materials and methods}

Experimental cell lines. Human hepatoma Hep3B and Huh7 cell lines were obtained from the American Type Culture Collection (Manassas, VA, USA). The MHCC97H cell line was gifted by Shanghai Zhongshan Hospital (Shanghai, China). All cell lines were maintained in DMEM with $10 \% \mathrm{FBS}$, $100 \mathrm{IU} / \mathrm{ml}$ penicillin $\mathrm{G}$ and $100 \mu \mathrm{g} / \mathrm{ml}$ streptomycin at $37^{\circ} \mathrm{C}$ in a $5 \% \mathrm{CO} 2$ incubator. Three pairs of HCSC sublines (Huh7-C, Hep3B-C and MHCC97H-C), which were previously demonstrated to show the reinforcement of stem cell properties, induction of drug resistance, an emergence of EMT properties, and enhancement of tumor-initiating capabilities (9), were derived from their case-matched human HCC cells (Hep3B, Huh7 and MHCC97H) and cultured in serum-free media with DMEM/F12 50\%, Neurobasal ${ }^{\mathrm{TM}}$-A Medium 50\%, B-27 Supplement Minus Vitamin A, EGF $20 \mu \mathrm{g} / \mathrm{ml}$, FGF-10 $20 \mu \mathrm{g} / \mathrm{ml}$, IGF-1 $20 \mu \mathrm{g} / \mathrm{ml}$, heparin $20 \mu \mathrm{g} / \mathrm{ml}, \beta-M E 20 \mu \mathrm{g} / \mathrm{ml}$, NEAA, BSA $0.25 \%$, GlutaMAX ${ }^{\mathrm{TM}}-1$. The study assembled all the aforementioned human HCC cell lines for RNA sequencing (RNA-Seq; Table I).

RNA-seq. RNA-seq was used to profile genes differentially expressed in cancer stem cells and their parental cell lines, Hep3B, Huh7 and MHCC97H. RNA-seq was performed by the Beijing Genomics Institute (BGI, Beijing, China). Total RNA was extracted using Eastep ${ }^{\text {TM }}$ Super Total RNA Extraction kit (Promega Corporation, Madison, WI, USA) and treated with DNase I. Subsequently, mRNA from total RNA was enriched with oligo (dT) magnetic beads for eukaryotes, followed by fragmentation into 200 bp short fragments by mixing with Fragmentation Reagents (Beckman Coulter, Inc., Danvers, MA, USA). Random hexamer priming was used to synthesize the first strand of cDNA; DNA polymerase I, dNTPs and RNase $H$ were used to synthesize the second strand; the double-stranded cDNA was purified with magnetic beads; and end repair and 3 '-end single nucleotide A addition were performed. Next, the adapter-modified fragments were enriched via PCR amplification (13). To monitor the quantity and quality of the DNA sample library, a quality control procedure was performed with an Agilent 2100 Bioanalyzer (Agilent Technologies, Inc., Santa Clara, CA, USA) and an ABI StepOnePlus RT-PCR system (Thermo Fisher Scientific, Inc., Waltham, MA, USA). The captured DNA library was sequenced to a depth of 30-fold coverage with the Illumina HiSeq ${ }^{\mathrm{TM}} 2000$ Analyzer (Illumina, Inc., San Diego, CA, USA).

Raw sequence preprocessing. To obtain high-quality reads for further analysis, data filtering was performed on the basis of adaptor sequences to remove low-quality reads, which were
Table I. Details of datasets from HCSCs and case-matched cells for RNA sequencing.

\begin{tabular}{llll}
\hline Data & \multicolumn{1}{c}{ Cells } & Total reads & Normalized \\
\hline RNA & Hep3B-C & 5762058 & RPKM \\
& Huh7-C & 5886142 & \\
& MHCC97H-C & & \\
& Hep3B & 6116344 & \\
& Huh7 & 5998659 & \\
MHCC97H & & \\
\hline
\end{tabular}

HCSCs, hepatic cancer stem-like cells.

defined by a percentage of unknown bases $(\mathrm{N})>10 \%$ or the presence of $>50 \%$ of bases with a quality value of 5 or less in a read. The clean reads were mapped to reference genomes and sequences with SOAP software (14). To exclude the effect of sequencing discrepancies and various gene lengths, gene expression levels were normalized using RPKM algorithms (reads per kb per million reads), making the transcripts across multiple samples comparable (15).

Data mining. The differentially expressed genes (DEGs) between the HCSCs and case-matched hepatic cancer cells were analyzed by the fold-change method (cut-off $>2$ or cut-off $<0.5$ ). Subsequently, genes with coherent differences in expression (consistently up- or downregulated in Huh7-C, Hep3B-C and MHCC97H-C cells, referred to as meta-DEGs) were selected, and the overlapping number of genes among the three cell line datasets was calculated using the Venny tool and illustrated in a Venn diagram. Hierarchical gene clustering was conducted using Cluster 3.0 software (16). The Euclidean distance similarity metric and the average linkage clustering method were used to measure gene patterns. TreeView $\mathrm{X}$ version 0.5.1 software (Slashdot Media, San Diego, CA, USA) was used to generate the clustering map.

Enrichment analysis was performed to study the functions and mechanisms of the DEGs. The OntoExpression tool was used to identify significantly enriched gene functions in the Gene Ontology (GO) database (http://geneontology.org/). Fisher's exact test was used for GO analysis. Gene Ontology functional classification was performed using WEGO software (17) to classify GO functions for DEGs. To identify the known pathways with significant enrichment with DEGs in HCSCs, pathway enrichment analysis was performed using Pathway-Express software in the Kyoto Encyclopedia of Genes and Genomes (KEGG) database (18). A hypergeometric test for the statistical scenario was used to screen GO terms or pathways that were significantly enriched in DEGs compared with the genomic background. The candidate GO terms or pathways with corrected P-values of $<0.05$ were considered to be significantly enriched.

miRNA target prediction. To analyze the interaction between miRNAs and genes, the results from 6 existing miRNA-target prediction programs including DIANA-microTv4.0 (19), miRanda-rel2010 (20), miRDB4.0 (21), miRWalk2.0 (22), 

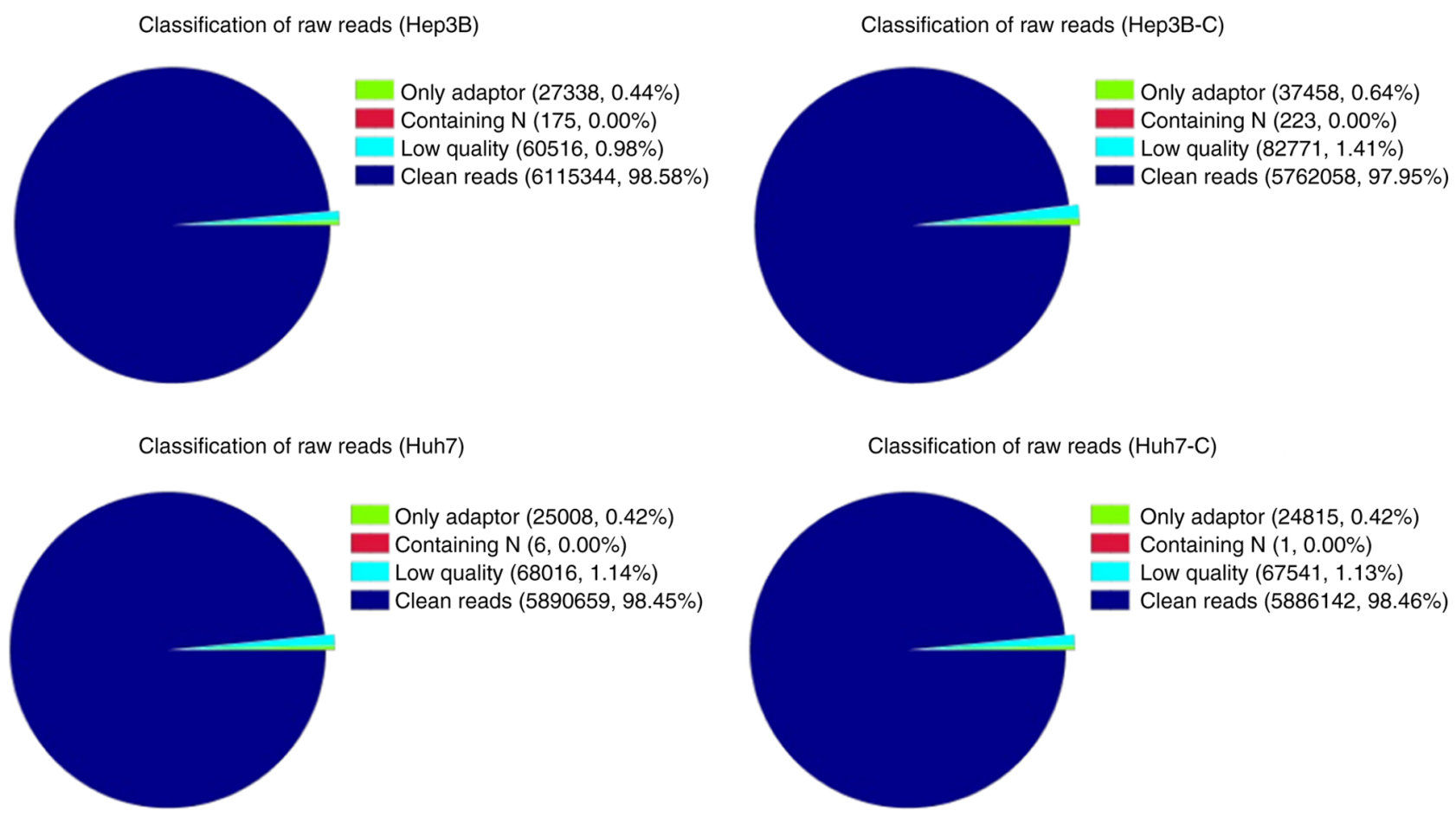

Classification of raw reads (MHCC97-H)

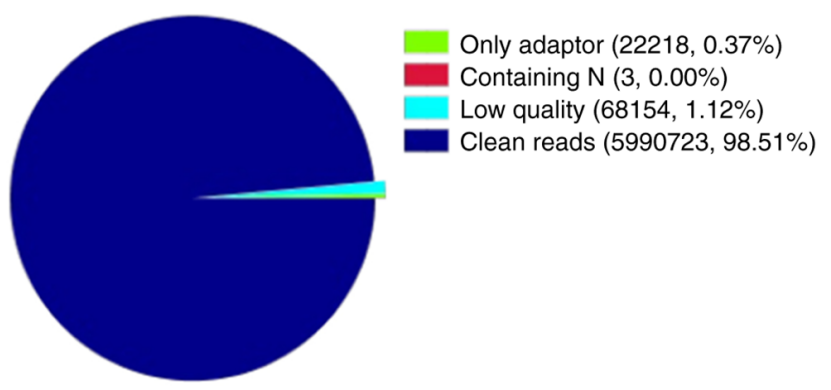

Classification of raw reads (MHCC97- $\mathrm{H}-\mathrm{C})$

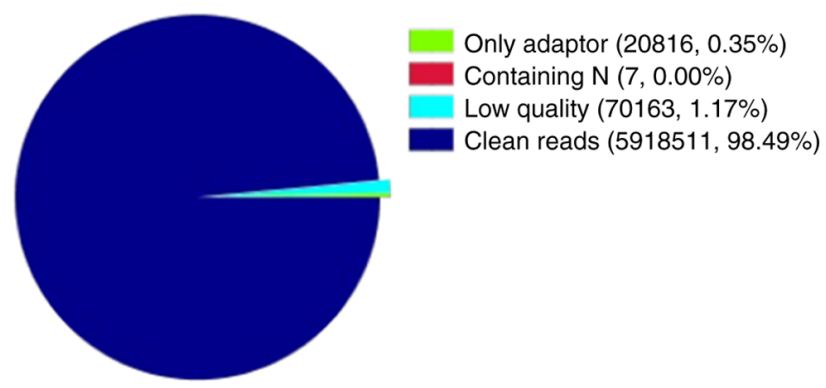

Figure 1. Classification of raw reads in six samples. Quality assessment was performed to demonstrate the composition of raw reads in Hep3B, Huh7, MHCC97-H, Hep3B-C, Huh7-C and MHCC97-H-C samples. For example, Only Adaptor (N, M\%) means that the number of reads containing adaptors is N, and the proportion is $\mathrm{M} \%$ of the total reads.

RNAhybrid2.1 (23), PicTar4 (24), PITA (25), RNA22v2 (26) and Targetscan6.2 (27), were compared. Candidate paired miRNAs and DEGs with at least five sources were considered for further analysis.

miRNA expression detection. The differential expression of miRNAs in cancer stem cells and their parental cell lines was detected by RT-qPCR (reverse transcription-quantitative polymerase chain reaction) using Eastep ${ }^{\mathrm{TM}}$ RT Master Mix (5X) (Promega, Madison, WI, USA).

Experimental validation. Perl scripts were run to conduct text-mining searches in the Entrez PubMed database.

Statistical analysis. Statistical analyses were performed using GraphPad Prism5 and R software version 3.2.1 (http://www.r-project.org/). Data are presented as the mean \pm standard deviation of the mean from at least three independent experiments. Statistically significant differences were calculated using Student's t-test and Kaplan-Meier survival analyses. Fisher's exact test was used to analyze the mRNA expression levels in hepatocellular carcinoma cells and corresponding hepatocellular carcinoma stem-like cells. ${ }^{*} \mathrm{P}<0.05 ;{ }^{* *} \mathrm{P}<0.01 ;{ }^{* * * *} \mathrm{P}<0.001$.

\section{Results}

Data and primary analysis. A data preprocessing procedure was performed for all samples to obtain high-quality reads, which demonstrated the classification of raw reads (Fig. 1). The proportion of clean reads was $>97.9 \%$ of the total reads in all the detected samples, indicating the existence of high-quality sequences following data filtering, upon which all subsequent analyses were based. Venn analysis with domestic code was applied to the three differentially expressed gene sets (DEGs according to the criteria: Of $\log 2 \mathrm{FC}>1$ or $<-1)$, and 1,012 DEGs were extracted from sphere-forming cells compared with the parental cells. Among these 1,012 DEGs satisfying the criterion of a fold-change of $>2$ or $<0.5,406$ DEGs overlapped with the consistent expression patterns among the three cell lines, 
A

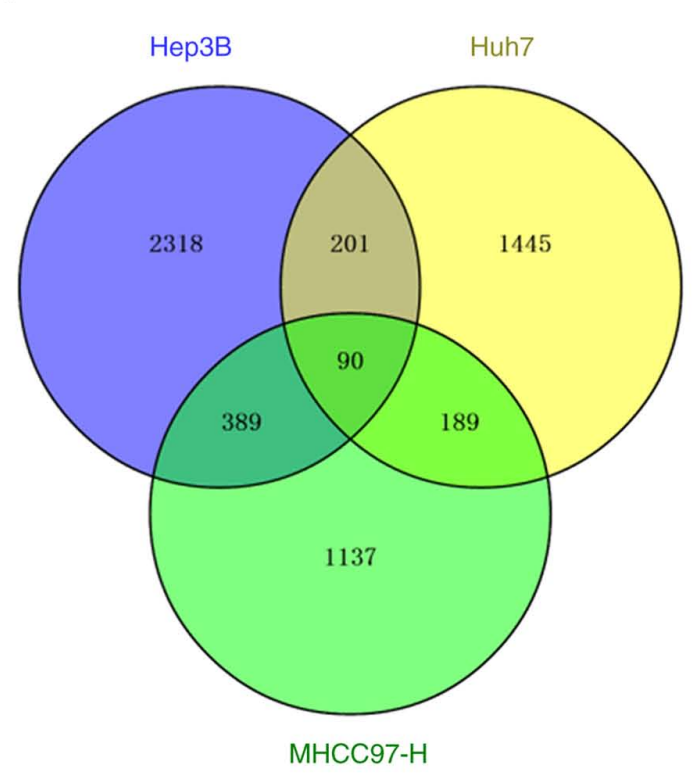

Down-regulated DEGs

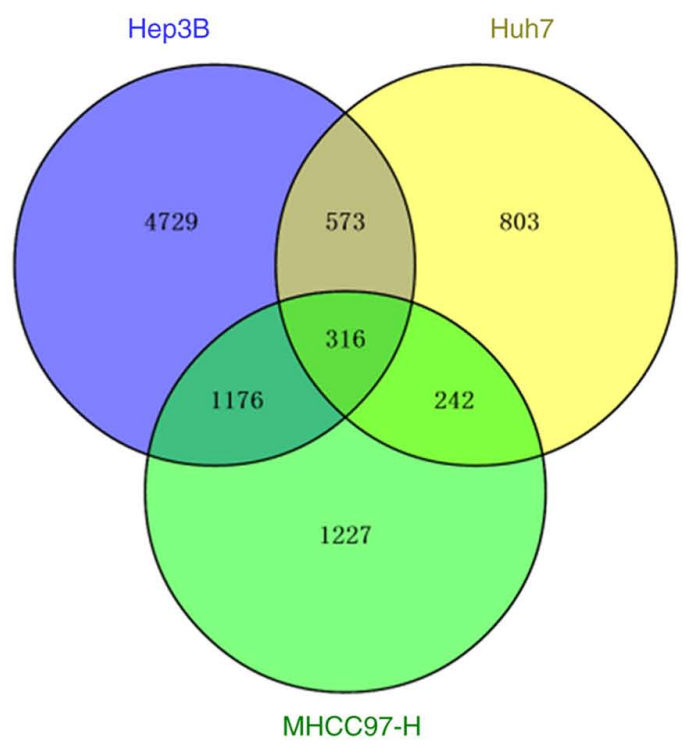

$\mathrm{B}$

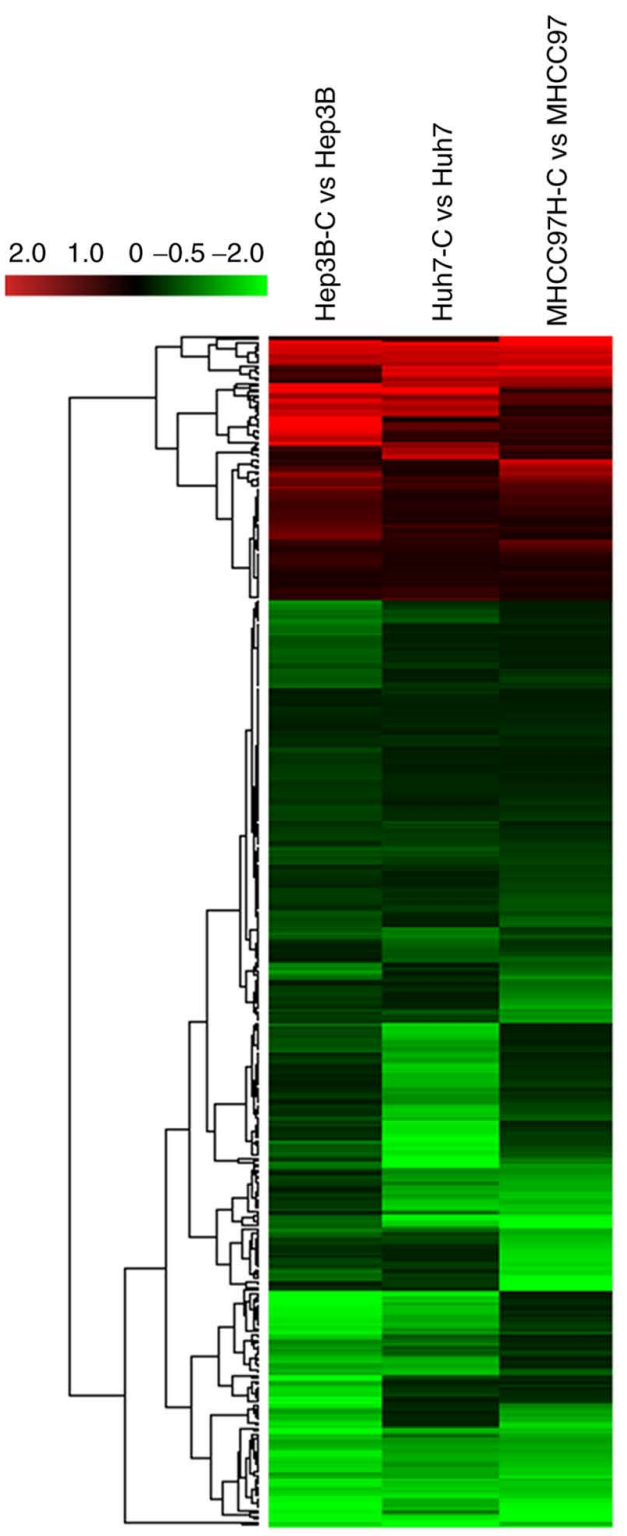

Figure 2. DEGs and their clustering map in 3 pairs of cell samples. (A) The identified DEGs are shown in a Venn diagram. In total, 406 DEGs overlapped in the 3 hepatic cancer stem-like cell lines compared with case-matched human hepatocellular carcinoma cells, among which 90 DEGs were upregulated and 316 were downregulated. (B) The clustering map shows all 406 significant DEGs identified in sphere-forming cells and their parental cells. Each column represents an experimental condition (e.g. Hep3B-C vs. Hep3B), and each row represents a gene. Expression differences are shown in different colors. Red corresponds to upregulation and green indicates downregulation. DEGs, differentially expressed genes.

including 90 DEGs that were upregulated and 316 DEGs that were downregulated in all three cell-lines (Fig. 2A). Similar expression patterns and functional correlations of these DEGs were also displayed (Fig. 2B).

GO functional classification and distribution of DEGs. To investigate the underlying mechanisms of the HCSCs, 406 DEGs were retrieved and their associations with HCSCs were investigated through analyzing GO and pathway enrichment. The WEGO tool was used to visualize the GO terms in molecular function, cellular component and biological process categories, which revealed the GO functional classification and distribution for the 406 DEGs (Fig. 3). The 7 most highly enriched terms for each level are indicated with blue color (corrected P-value <0.05), and 39, 8, and 26 GO terms were significantly enriched in the molecular function, cellular component and biological process categories, respectively, with corrected P-values of $<0.05$ (Tables SI-III).

KEGG pathways enriched with DEGs. There were 53 pathways enriched with DEGs, including the complement and coagulation cascade pathway, the peroxisome proliferator-activated receptor (PPAR) signaling pathway, the extracellular matrix (ECM)-receptor interaction pathway, and the transforming growth factor (TGF)-beta signaling pathway. However, only the complement and coagulation cascade pathways 


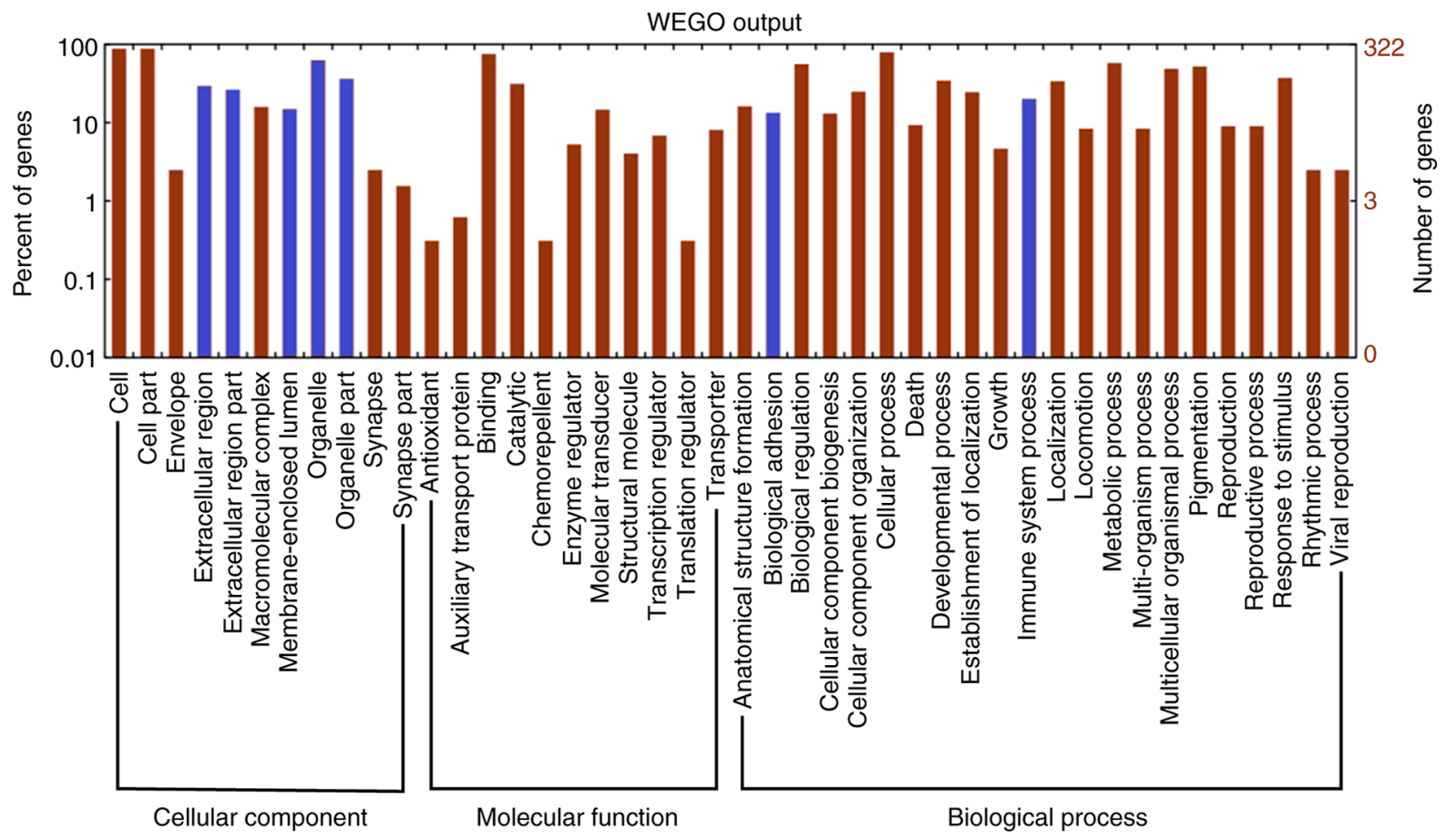

Figure 3. Gene ontology annotations for the DEGs of the predicted hepatic cancer stem-like cell biomarkers. The identified 406 DEGs were retrieved and annotated with WEGO software in three Gene Ontology domains: Molecular function, biological process and cellular component. The 7 most highly enriched terms for each domain are indicated in blue. DEGs, differentially expressed genes.

reached statistical significance according to Pathway-Express (corrected P-value $=0.0330$, P-value $=0.0006$; Table II). It was also found that the DEGs were enriched in ECM-receptor interaction, the PPAR signaling pathway and the TGF- $\alpha$ signaling pathway associated with signaling transduction, although none of the corresponding corrected $\mathrm{P}$-values reached statistical significance, as calculated by false discovery rate (FDR) correction.

Functional analysis of complement-related DEGs. As expected, several DEGs were also enriched in the GO term of complement activation, with a corrected P-value of 0.0260 (Fig. 4A). The scatterplots comparing the deviation of DEGs between HCSC samples and case-matched hepatic cancer cell samples are shown, in which 7 complement-related DEGs including complement component 1s (C1S), complement component 1r (C1R), complement factor I (CFI), C3, serpin family A member 5 (SERPINA5), serpin family $\mathrm{G}$ member 1 (SERPING1) and kininogen 1 (KNG1), are indicated with blue dots (Fig. 4B). Clustering analysis was also integrated to obtain the expression patterns of 7 DEGs involved in complement activation (Fig. 4C).

Prediction of miRNA target networks involving complement-related DEGs. The associations between the enriched pathways were investigated via text mining searches in NCBI PubMed. Notably, the complement and coagulation cascade pathways were found to be implicated in the development of HCSCs, which has been investigated previously in HCC. However, the downregulation of these pathways has not been described in HCSCs. In our previous study (9), the analysis of miRNAs and target genes was integrated to study the molecular signatures and regulatory mechanisms in HCSCs. Through network analysis, miRNA target networks were constructed. In the present study, twenty miRNAs were predicted to be candidates involved in complement activation (Table III). Among these miRNAs, 7 (4 up- and 3 downregulation) had previously been shown to be differentially expressed by miRNA high-throughput sequencing. It was predicted that hsa-miR-186 and hsa-miR-187 may regulate C1S, hsa-miR-197 may interact with CFI, and SERPINA5 was targeted by hsa-miR-183, hsa-miR-450b and hsa-miR-532 (Fig. 5).

\section{Discussion}

To clarify the carcinogenic mechanisms of HCC to investigate effective strategies to treat HCC, our previous studies provided evidence of the establishment of a cell culture system that allowed the culture and amplification of HCSCs (9). Subsequently, a large-scale investigation was performed encompassing whole-genome transcriptomic profiling, analysis for pathways, gene ontologies and miRNA-target interaction predictions to investigate, not only the gene expression alteration of HCSCs, but also the signaling pathways involving these DEGs as well as the potential roles of HCSCs.

A total of 406 significantly DEGs were identified and stratified according to the selected criteria. An enrichment analysis was performed using the publicly available GO and KEGG datasets to investigate HCSC regulatory mechanisms. Seventy-three GO terms were found to be significantly associated with genes that were up- or downregulated in HCSCs (corrected P-value <0.05). Next, the enrichment of DEGs were searched for in pathways through Pathway-Express analysis. Only complement and coagulation cascade pathways were 
Table II. Significantly enriched Kyoto Encyclopedia and Genes and Genomes pathways.

\begin{tabular}{lcccc}
\hline Pathway name & Input & Reference & P-value & Corrected P-value \\
\hline Complement and coagulation cascades & 7 & 62 & 0.000617 & 0.032717195 \\
ECM-receptor interaction & 7 & 77 & 0.002234 & 0.059193855 \\
PPAR signaling & 5 & 63 & 0.016303 & 0.28801761 \\
TGF-beta signaling & 5 & 0.044279 & 0.586702115
\end{tabular}

$\mathrm{P}<0.05$ was considered to indicate a statistically significant difference, using Fisher's exact test. ECM, extracellular matrix; PPAR, peroxisome proliferator-activated receptor; TGF, transforming growth factor.

A

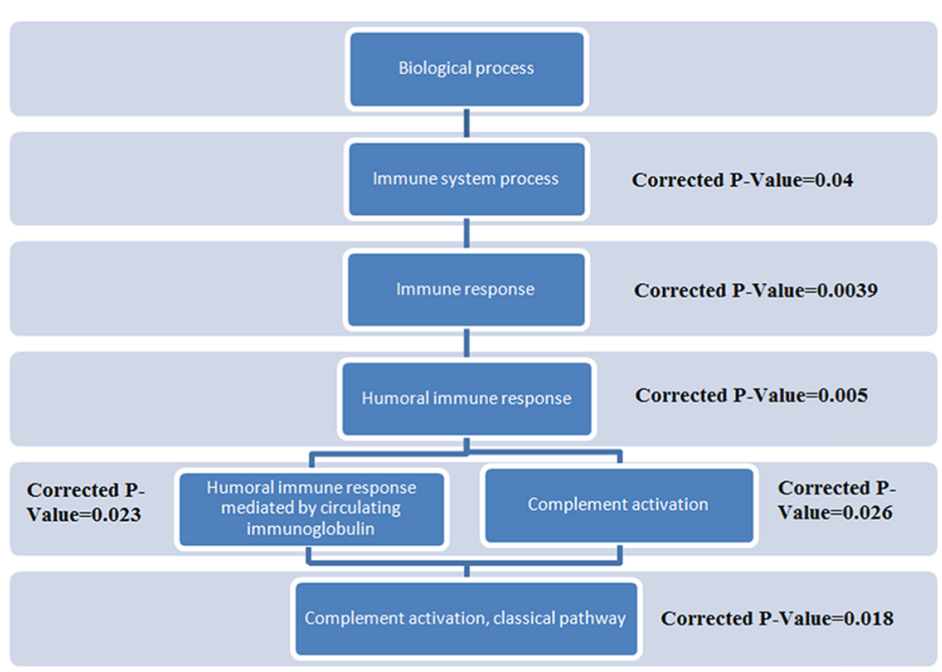

C

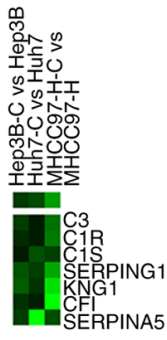

B
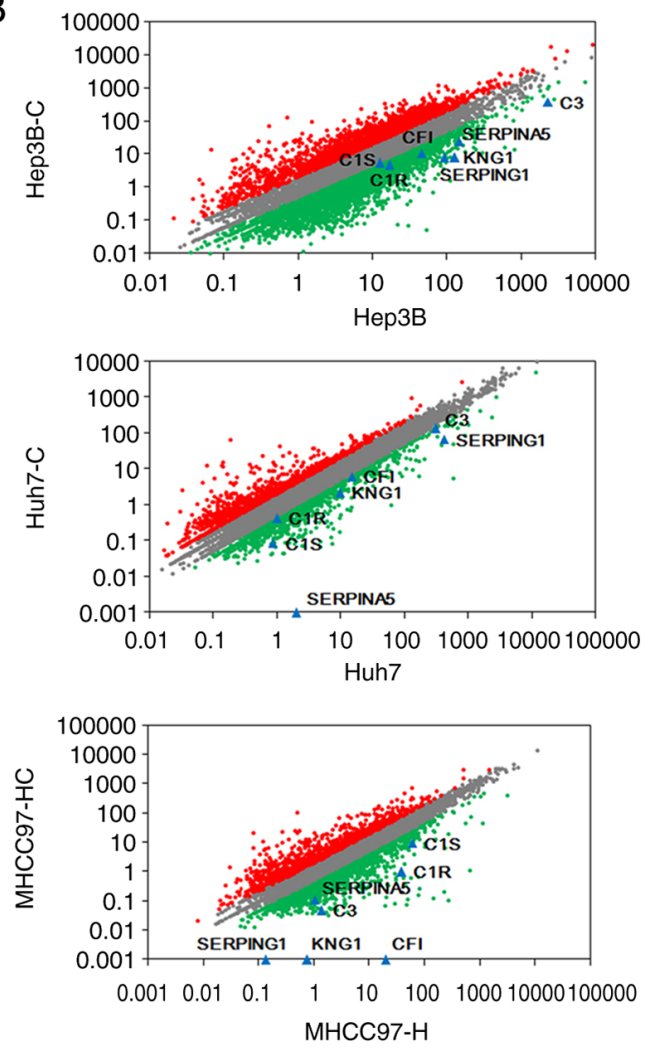

Figure 4. Functional analysis of complement-related DEGs (differentially expressed genes). (A) The term subordination relationship of immune system process in GO (gene ontology) analysis. The subordination relationships of the GO terms are reflected by their positions in the figure. Corrected P-values are indicated in the figure. (B) Scatter plots of global gene expression patterns and 7 DEGs. The scatter plots describe global gene expression patterns between hepatic cancer stem cells and human hepatocellular carcinoma samples. Red, grey and green represent gene expression showing upregulated, equivalent- and downregulated levels. The positions of C1S, C1R, CFI, C3, SERPINA5, SERPING1 and KNG1 are indicated with blue dots. (C) Two-way hierarchical clustering showing the 7 complement activation-related genes. C1S, complement component 1s; CR1, complement component 1r; CFI, complement factor I; SERPINA5, serpin family A member 5; SERPING1, serpin family G member 1; KNG1, kininogen 1.

identified as significantly enriched pathways (corrected P-value $<0.05)$. Gene expression studies have previously implicated these pathways in HCC (28-30). These results revealed the upregulation of the complement and coagulation cascade pathways in HCC cases versus patients with liver cirrhosis. Recently, the upregulated expression of mCRPs (membrane-bound complement restriction proteins) was reported to sequester cancer cells from complement-dependent cytotoxicity and enhance the survival ability of cancer cells in head and neck cancer (31). However, the downregulation of complement and coagulation cascade pathways has not been described previously in HCSCs. By combining the results from the GO and
KEGG analyses, 7 genes were found to be downregulated in the complement and coagulation cascade pathways (C1S, C1R, CFI, C3, SERPINA5, SERPING1 and KNG1). These genes were previously confirmed to serve an important role in cancer. For example, CFI is overexpressed in cutaneous squamous cell carcinoma progression (32) and is upregulated in breast cancer and involved in poor clinical outcomes (33). CFI is a potential suppressive protein in gastric cancer (34), and both CFI and KNG1 show differential expression compared with their levels in HBV-infected and healthy controls, which suggests that there is a possible association between these genes and HCC progression (35). Additionally, SERPINA5 serves an important role in 
Table III. Details of differentially expressed genes regulated by predicted miRNAs.

\begin{tabular}{|c|c|c|c|}
\hline Gene name & MicroRNA & SUM & CHIP \\
\hline \multirow[t]{5}{*}{$\mathrm{C} 1 \mathrm{~S}$} & hsa-miR-186 & 6 & DOWN \\
\hline & hsa-miR-187 & 5 & DOWN \\
\hline & hsa-miR-501-5p & 6 & \\
\hline & hsa-miR-580 & 6 & \\
\hline & hsa-miR-634 & 5 & \\
\hline \multirow[t]{3}{*}{ CFI } & hsa-miR-197 & 5 & UP \\
\hline & hsa-miR-412 & 5 & \\
\hline & hsa-miR-580 & 6 & \\
\hline \multirow[t]{12}{*}{ SERPINA5 } & hsa-miR-138 & 5 & \\
\hline & hsa-miR-183 & 5 & UP \\
\hline & hsa-miR-205 & 6 & \\
\hline & hsa-miR-211 & 5 & \\
\hline & hsa-miR-31 & 6 & \\
\hline & hsa-miR-338-3p & 5 & UP \\
\hline & hsa-miR-376a & 5 & \\
\hline & hsa-miR-450b-5p & 6 & UP \\
\hline & hsa-miR-532-3p & 5 & DOWN \\
\hline & hsa-miR-636 & 5 & \\
\hline & hsa-miR-643 & 6 & \\
\hline & hsa-miR-649 & 6 & \\
\hline
\end{tabular}

C1S, complement component S1; CFI, complement factor I; SERPINA5, serpin family A member 5.

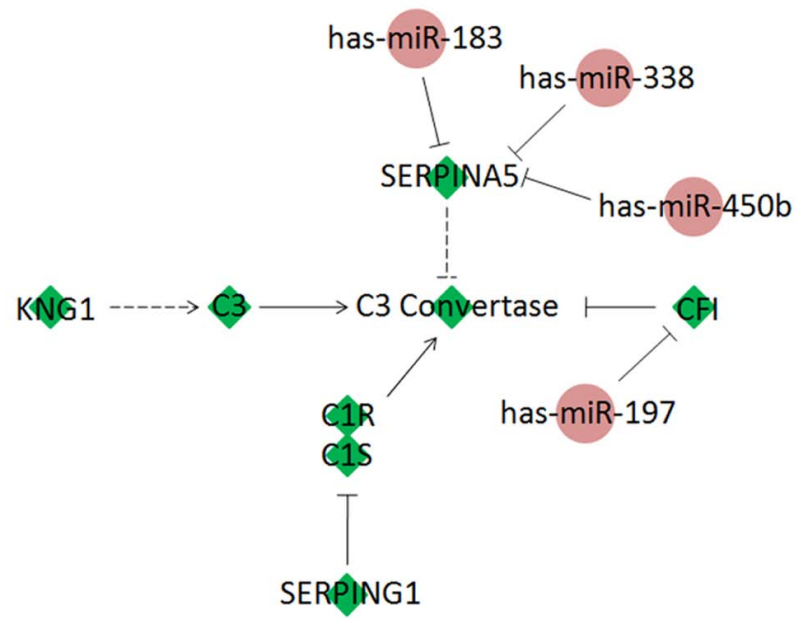

Figure 5. Differentially expressed miRNAs involved in complement and coagulation cascade networks. Among the miRNAs that were differentially expressed according to miRNA high-throughput sequencing, it was predicted that hsa-miR-197 interacted with CFI, and SERPINA5 was targeted by hsa-miR-183, hsa-miR-338 and hsa-miR-450b. CFI, complement factor I; SERPINA5, serpin family A member 5 .

the regulation of HCC migratory and metastatic potentials (36). Our RNA-seq results demonstrated that the expression of C1S, C1R, CFI, C3, SERPINA5, SERPING1 and KNG1 was significantly different between carcinoma stem-like cells and tumor cells. All the results are presented in Table SIV.
The miRNA-mRNA network constructed in the present study consisted of 7 complement activation related genes and their computationally predicted associated miRNAs. The data resources of the predictive computation databases were derived from the comparison of binding sites in 12 existing miRNA-target predictive programs. Seven miRNAs were predicted to target several downregulated genes involved in the complement activation pathway. C1S was demonstrated to bind the putative binding sites for hsa-miR-186 and hsa-miR-187, and hsa-miR-197 was found to bind the 3'-UTR of CFI. Similarly, SERPINA5 was identified to contain binding sites for several miRNAs, including hsa-miR-183, hsa-miR-450b and hsa-miR-532.

The expression of miR-338 and miR-450b in Hep3B, Hep3B-C, Huh7 and Huh7-C cells was detected by reverse transcription-quantitative polymerase chain reaction. The results showed that the expression of miR-338 and miR-450b was significantly increased in HCSCs (Fig. S1). Notably, hsa-miR-450b-5p was also identified in our previous study, which was predicted to participate in HCSC invasionrelated pathways. The mitogen-activated protein kinase (MAPK)/extracellular signal-regulated kinase (ERK) signaling pathway was enriched with several target genes of hsa-miR-450b-5p. A recent study involving a pluripotent stem cell model revealed the essential role of miR-450b-5p in embryonic corneal lineage specification (37). However, the regulation of miR-450b-5p as a molecular switch of SERPINA5 involved in the complement and coagulation cascade pathways has not yet been investigated.

In conclusion, the present analysis, together with mRNA and miRNA high-throughput sequencing data, revealed novel distinctive signatures of HCSCs. The complement and coagulation cascade pathways were identified during the development of HCSCs, which may contribute toward hepatic cancer stem cell studies and the identification of new drugs for the treatment of HCC. However, this study only performed RNA-seq on three hepatocellular carcinoma cell lines, and no study was conducted on fresh HCC tissue samples. The conclusions of the present study need to be further confirmed by a broader investigation.

\section{Acknowledgements}

Not applicable.

\section{Funding}

The present study was supported by the National Key R\&D Program of China (grant no. 2018YFA0900900) and the National Natural Science Foundation of China (grant nos. 81802736 and 81773251).

\section{Availability of data and materials}

All data generated or analyzed during this study are included in this published article.

\section{Authors' contributions}

$\mathrm{JL}$ and $\mathrm{KL}$ designed and performed all the experiments. JL, KL, YS, QZ, LC, HQ and HW participated in the acquisition 
and analysis of data. JL and CS drafted and revised the article. CS designed the experiments and agreed to be accountable for all aspects of the work in ensuring that questions related to the accuracy or integrity of any part of the work are appropriately investigated and resolved. All authors read and approved the final manuscript.

\section{Ethics approval and consent to participate}

Not applicable.

\section{Patient consent for publication}

Not applicable.

\section{Competing interest}

The authors declare that they have no competing interests.

\section{References}

1. Siegel RL, Miller KD and Jemal A: Cancer statistics, 2020. CA Cancer J Clin 70: 7-30, 2020.

2. Villanueva A, Minguez B, Forner A, Reig M and Llovet JM: Hepatocellular carcinoma: Novel molecular approaches for diagnosis, prognosis, and therapy. Annu Rev Med 61: 317-328, 2010.

3. Yang JD and Roberts LR: Hepatocellular carcinoma: A global view. Nat Rev Gastroenterol Hepatol 7: 448-458, 2010.

4. Yau T, Chan P, Epstein R and Poon RT: Evolution of systemic therapy of advanced hepatocellular carcinoma. World J Gastroenterol 14: 6437-6441, 2008.

5. Chun JM, Kwon HJ, Sohn J, Kim SG, Park JY, Bae HI, Yun YK and Hwang YJ: Prognostic factors after early recurrence in patients who underwent curative resection for hepatocellular carcinoma. J Surg Oncol 103: 148-151, 2011.

6. Guo Z, Li LQ, Jiang JH, Ou C, Zeng LX and Xiang BD: Cancer stem cell markers correlate with early recurrence and survival in hepatocellular carcinoma. World J Gastroenterol 20: 2098-2106, 2014.

7. Ma S, Lee TK, Zheng BJ, Chan KW and Guan XY: CD133+ HCC cancer stem cells confer chemoresistance by preferential expression of the Akt/PKB survival pathway. Oncogene 27 $1749-1758,2008$

8. Sell S and Leffert HL: Liver cancer stem cells. J Clin Oncol 26: 2800-2805, 2008.

9. Li J, Yu Y, Wang J, Yan Z, Liu H, Wang Y, Ding M, Cui L, Wu M, Jiang X and Qian Q: Establishment of a novel system for the culture and expansion of hepatic stem-like cancer cells. Cancer Lett 360: 177-186, 2015.

10. Yang ZF, Ho DW, Ng MN, Lau CK, Yu WC, Ngai P, Chu PW, Lam CT, Poon RT and Fan ST: Significance of CD90+ cancer stem cells in human liver cancer. Cancer Cell 13: 153-166, 2008.

11. Yamashita T, Forgues M, Wang W, Kim JW, Ye Q, Jia H, Budhu A, Zanetti KA, Chen Y, Qin LX, et al: EpCAM and alpha-fetoprotein expression defines novel prognostic subtypes of hepatocellular carcinoma. Cancer Res 68: 1451-1461, 2008.

12. Szeto CY, Lin CH, Choi SC, Yip TT, Ngan RK, Tsao GS and Li Lung M: Integrated mRNA and microRNA transcriptome sequencing characterizes sequence variants and mRNA-microRNA regulatory network in nasopharyngeal carcinoma model systems. FEBS Open Biol 4: 128-140, 2014.

13. Lin T, Liu Q and Chen J: Identification of differentially expressed genes in Monochamus alternatus digested with azadirachtin. Sci Rep 6: 33484, 2016.

14. Li R, Li Y, Kristiansen K and Wang J: SOAP: Short oligonucleotide alignment program. Bioinformatics 24: 713-714, 2008.

15. Mortazavi A, Williams BA, McCue K, Schaeffer L and Wold B: Mapping and quantifying mammalian transcriptomes by RNA-Seq. Nat Methods 5: 621-628, 2008.

16. de Hoon MJ, Imoto S, Nolan J and Miyano S: Open source clustering software. Bioinformatics 20: 1453-1454, 2004.

17. Ye J, Zhang Y, Cui H, Liu J, Wu Y, Cheng Y, Xu H, Huang X, Li S, Zhou A, et al: WEGO 2.0: A web tool for analyzing and plotting GO annotations, 2018 update. Nucleic Acids Res 46: W71-W75, 2018.
18. Kanehisa M, Araki M, Goto S, Hattori M, Hirakawa M, Itoh M, Katayama T, Kawashima S, Okuda S, Tokimatsu T and Yamanishi Y: KEGG for linking genomes to life and the environment. Nucleic Acids Res 36 (Database Issue): D480-D484, 2008.

19. Maragkakis M, Vergoulis T, Alexiou P, Reczko M, Plomaritou K, Gousis M, Kourtis K, Koziris N, Dalamagas T and Hatzigeorgiou AG DIANA-microT Web server upgrade supports fly and worm miRNA target prediction and bibliographic miRNA to disease association. Nucleic Acids Res 39 (Web Server Issue): W145-W148, 2011.

20. John B, Enright AJ, Aravin A, Tuschl T, Sander C and Marks DS: Human MicroRNA targets. PLoS Biol 2: e363, 2004.

21. Wang X: miRDB: A microRNA target prediction and functional annotation database with a wiki interface. RNA 14: 1012-1017, 2008

22. Dweep H, Sticht C, Pandey P and Gretz N: miRWalk-database: Prediction of possible miRNA binding sites by 'walking' the genes of three genomes. J Biomed Inform 44: 839-847, 2011.

23. Rehmsmeier M, Steffen P, Hochsmann M and Giegerich R: Fast and effective prediction of microRNA/target duplexes. RNA 10: 1507-1517, 2004

24. Krek A, Grün D, Poy MN, Wolf R, Rosenberg L, Epstein EJ, MacMenamin P, da Piedade I, Gunsalus KC, Stoffel M and Rajewsky N: Combinatorial microRNA target predictions. Nat Genet 37: 495-500, 2005

25. Kertesz M, Iovino N, Unnerstall U, Gaul U and Segal E: The role of site accessibility in microRNA target recognition. Nat Genet 39: 1278-1284, 2007.

26. Miranda KC, Huynh T, Tay Y, Ang YS, Tam WL, Thomson AM, Lim B and Rigoutsos I: A pattern-based method for the identification of MicroRNA binding sites and their corresponding heteroduplexes. Cell 126: 1203-1217, 2006.

27. Lewis BP, Burge CB and Bartel DP: Conserved seed pairing, often flanked by adenosines, indicates that thousands of human genes are microRNA targets. Cell 120: 15-20, 2005.

28. Tsai TH, Song E, Zhu R, Di Poto C, Wang M, Luo Y, Varghese RS, Tadesse MG, Ziada DH, Desai CS, et al: LC-MS/MS-based serum proteomics for identification of candidate biomarkers for hepatocellular carcinoma. Proteomics 15: 2369-2381, 2015.

29. Zhang L, Guo Y, Li B, Qu J, Zang C, Li F, Wang Y, Pang H, $\mathrm{Li} \mathrm{S}$ and Liu Q: Identification of biomarkers for hepatocellular carcinoma using network-based bioinformatics methods. Eur J Med Res 18: 35, 2013.

30. Lv J, Zhu B, Zhang L, Xie Q and Zhuo W: Detection and screening of small molecule agents for overcoming Sorafenib resistance of hepatocellular carcinoma: A bioinformatics study. Int J Clin Exp Med 8: 2317-2325, 2015.

31. Kesselring R, Thiel A, Pries R, Fichtner-Feigl S, Brunner S, Seidel P, Bruchhage KL and Wollenberg B: The complement receptors CD46, CD55 and CD59 are regulated by the tumour microenvironment of head and neck cancer to facilitate escape of complement attack. Eur J Cancer 50: 2152-2161, 2014.

32. Riihilä P, Nissinen L, Farshchian M, Kivisaari A, Ala-Aho R, Kallajoki M, Grénman R, Meri S, Peltonen S, Peltonen J and Kähäri VM: Complement factor I promotes progression of cutaneous squamous cell carcinoma. J Invest Dermatol 135: $579-588,2015$

33. Okroj M, Holmquist E, Nilsson E, Anagnostaki L, Jirström K and Blom AM: Local expression of complement factor I in breast cancer cells correlates with poor survival and recurrence. Cancer Immunol Immunother 64: 467-478, 2015.

34. Liu W, Liu B, Xin L, Zhang Y, Chen X, Zhu Z and Lin Y: Down-regulated expression of complement factor I: A potential suppressive protein for gastric cancer identified by serum proteome analysis. Clin Chim Acta 377: 119-126, 2007.

35. He X, Wang Y, Zhang W, Li H, Luo R, Zhou Y, Liao CL, Huang H, $\mathrm{Lv} \mathrm{X}, \mathrm{Xie} \mathrm{Z}$ and He M: Screening differential expression of serum proteins in AFP-negative HBV-related hepatocellular carcinoma using iTRAQ-MALDI-MS/MS. Neoplasma 61: 17-26, 2014.

36. Jing Y, Jia D, Wong CM, Oi-Lin Ng I, Zhang Z, Liu L, Wang Q, Zhao F, Li J, Yao M, et al: SERPINA5 inhibits tumor cell migration by modulating the fibronectin-integrin $\beta 1$ signaling pathway in hepatocellular carcinoma. Mol Oncol 8: 366-377, 2014.

37. Shalom-Feuerstein R, Serror L, De La Forest Divonne S, Petit I, Aberdam E, Camargo L, Damour O, Vigouroux C, Solomon A, Gaggioli C, et al: Pluripotent stem cell model reveals essential roles for miR-450b-5p and miR-184 in embryonic corneal lineage specification. Stem Cells 30: 898-909, 2012. 